\title{
Sleep Deprivation, EEG, and Functional MRI in Depression: Preliminary Results
}

\author{
Camellia P. Clark, M.D., Lawrence R. Frank, Ph.D., and Gregory G. Brown, Ph.D.
}

One night of total or partial sleep deprivation (SD) produces temporary remissions in 40-60\% of patients with major depression. Two unmedicated patients with major depression and a matched control received quantitative perfusion MR images at baseline and after one night of partial SD (PSD). A reduction $\geqslant 30 \%$ in the 17 -item Hamilton Depression Rating Scale (omitting sleep and weight loss items) defined antidepressant response. Theory, techniques, strengths and weaknesses of quantitative perfusion MRI are described in detail. At baseline, the responder exhibited elevated perfusion covering ventral anterior cingulate/medial frontal cortex; the control's maximal perfusion area was markedly smaller. The nonresponder's perfusion was lowest of all, particularly ventrally. PSD decreased perfusion over much of the responder's hyperperfused area but did not change the nonresponder's scan. These preliminary findings are consistent with previous SD studies using PET and SPECT.

[Neuropsychopharmacology 25:S79-S84, 2001] (C) 2001 American College of Neuropsychopharmacology. Published by Elsevier Science Inc.
KEY WORDS: Sleep deprivation; Depression; Functional MRI; Perfusion; Cingulate

\section{INTRODUCTION}

\section{Antidepressant Effects of Sleep Deprivation}

One of the most fascinating phenomena in all of biological psychiatry is the antidepressant effect of sleep deprivation (SD). Based on numerous studies including over 1700 patients over the past quarter century, (Wu and Bunney 1990) a single night of total or partial SD (PSD) produces clinically significant remissions in 40$60 \%$ of patients with major depression, including hundreds who were not taking medication. (Gillin 1983; Wu and Bunney 1990; Leibenluft and Wehr 1992). Sleep

From the University of California, San Diego and San Diego Veterans Affairs Medical Center, San Diego, CA.

Address correspondence to: Dr. Camellia P. Clark, Department of Psychiatry 9116A, University of California at San Diego, 9500 Gilman Dr., La Jolla, CA 92093, Tel.: (858) 552-8585x2580, Fax: (858) 458-4201, E-mail: cclark@vapop.ucsd.edu deprivation is an almost an ideal experimental model of an antidepressant therapy: (1) because of its rapid onset and offset, a patient can be studied within a 24-hour period before and after a clinically significant effect; (2) SD can circumvent the confounding and nonspecific effects of medications - a particular advantage for functional brain imaging; and (3) normal controls can undergo the same experimental procedures as patients.

The functional imaging findings associated with SD in depression are remarkably consistent. First, all studies using positron emission tomography (PET) with ${ }^{18} \mathrm{~F}-$ deoxyglucose (FDG) or single photon emission computed tomography (SPECT) with hexamethyl propylene-amine oxime (HMPAO) report at least one area in which responders and nonresponders differ significantly at baseline, with most studies reporting that baseline ventral anterior cingulate/basal orbital/medial frontal activity is greater in responders than in nonresponders. Second, all studies report differential response to SD in responders and nonresponders, with significantly reduced anterior cingulate cortex activity in responders but not in nonresponders (Wu et al. 1992; Ebert et al. 1991, 1994; Volk et al. 1992; Wu et al. 1999). 
Similar imaging findings, involving changes in the basal orbital/ medial frontal cortex, especially the anterior cingulate, have been linked with clinical response to medications (Mayberg et al. 1997; Buchsbaum et al. 1997).

\section{Functional Magnetic Resonance Imaging}

Functional magnetic resonance imaging (fMRI) features many practical and scientific advantages over PET and SPECT. Its lack of radioactivity and feasibility of implementation on clinical MRI scanners make it safe, inexpensive, and readily available to many hospitals. In addition to spatial resolution superior to PET and SPECT, fMRI demonstrates better temporal resolution because of the mechanisms of signal production and measurement (Ogawa et al. 1998). The purpose of this paper is to describe this new technique and present preliminary data on the first use ever of fMRI to study the antidepressant effects of SD.

MR methods of measuring cerebral perfusion, such as the pulsed arterial spin labeling (ASL) techniques used to obtain the images presented below, can be thought of as similar to other diffusible tracer imaging techniques (e.g., $\mathrm{H}_{2}{ }^{15} \mathrm{O}-\mathrm{PET}$ ); however, the "tracer" (magnetically tagged blood) half-life is roughly $1 \mathrm{~s}$ (the T1 of blood). Because of this extremely brief half-life, ASL is sensitive mainly to the rate of tagged blood delivery to the tissue; in contrast, $\mathrm{H}_{2}{ }^{15} \mathrm{O}$-PET is sensitive to exchange of $\mathrm{H}_{2}{ }^{15} \mathrm{O}$ into tissues and to its outflow as well as the rate of tracer delivery to the imaging slice (Wong et al. 1999).

Measuring perfusion using a pulsed arterial tag involves imaging a slice in alternating tagged and control states (Wong et al. 1997). Typically the tag involves inverting spins flowing into the imaging slice. The control state attempts to separate the non-specific effects of tagging from the effects produced by the perfusion of inverted spins into the slice. Blood flowing into the imaging slice will be positive in the difference image formed by subtracting the slice imaged obtained during the tagged state from the slice during the control state (Wong et al. 1997). Because of the short half-life of the magnetic "tracer" used, ASL methods primarily measure cerebral perfusion in capillary beds, with few tagged spins reaching venous vessels (Wong 1999). In contrast, the commonly used BOLD (blood oxygen level dependent) functional imaging method primarily represents signal from veins, which might be as much as $2 \mathrm{~cm}$ distant from the site of neuronal activity (Wong 1999). Even if one applies gradients to destroy intravenous signal, extravascular BOLD effects remain adjacent to veins. Furthermore, the BOLD signal represents a complex combination of local oxygen extraction, cerebral blood flow, and blood volume. Thus, unlike the perfusion signal, BOLD signal does not directly measure a well-defined biological parameter (Wong 1999). While relative changes in BOLD signal can be measured with reliability and precision, signal intensity for a comparison task or condition may vary between runs; even BOLD data from individual runs requires correction for "baseline drift." This variability represents a potential problem for within-subjects designs in which data from separate scanning sessions on separate days are compared.

\section{Potential Sources of Perfusion Measurement Error}

Any differences in local tissue magnetization within the image slice between tag and control conditions could affect a simple subtraction. Continuous and pulsed ASL methods (including QUIPSS (quantified imaging of perfusion using single subtraction) II) generally avoid this problem by carefully applying a saturation pulse (to destroy preexisting magnetic signal) to the imaging slice immediately before each tag and control condition.

Because the tagging region is not co-extensive with the image slice(s), one must account for the delay(s) before the tagged blood arrives. (Obviously, one must account for longer transit delays to more distal slices in multislice protocols as well, which requires careful measurements and kinetic modeling.) Typical transit delays in normal humans are on the same order of magnitude as the $\mathrm{T} 1$ of blood; too long a transit delay can introduce T1 decay into the image. This can be minimized by use of a tagged bolus of well-defined time width; once the whole bolus reaches the imaging slice, the ASL is directly proportional to the (known) tagging time width plus CBF. In QUIPSS II this proportionality is established by tagging with a slab-selective inversion pulse, waiting a delay "TI1" later, and then saturating the tagging region to destroy any remaining tag. The QUIPSS II method creates a bolus of tagged blood with a well-defined width of time TI1, and after waiting an additional time (typically about $700 \mathrm{msec}$ ) for the tagged bolus to reach the slice of interest, the image is acquired at time TI2. As long as (TI2-TI1) is longer than the longest transit delay from the tagging band to the slice, variations in transit delay will not affect the measured signal.

If any tag remains within an artery in the imaging slice, it is impossible to tell if that artery is perfusing that slice or "passing through" to perfuse a more distal site. While one can use bipolar gradient pulses to attenuate signal from flowing spins in large vessels, such pulses effectively create a longer transit delay, in the sense that the tagged spins will not be detectable until they reach the small vessels with slower flow (Wong 1999). The delay TI2-TI1 in QUIPSS II allows tagged spins in large vessels that do not feed a capillary bed in the voxel to pass on through so that they will not contribute to the signal.

As discussed above, the measured perfusion in QUIPSS II, based on the difference between tagged and 
control signal, is directly proportional to local CBF (Wong et al. 1997, 1998). Other factors entering in to the proportionality are $\mathrm{MOb}$ (the signal intensity of fully relaxed arterial blood) and a decay term that depends primarily on the T1 of blood. (With QUIPSS II, most of the tagged spins have not exchanged into tissue at the imaging time, so decay of the tag depends primarily on relaxation in blood.) In order to express our results in absolute units of $\mathrm{ml}$ blood per $\mathrm{ml}$ tissue per minute, we would have to estimate these blood MRI parameters, and this is not easy to do in practice. However, because these MR parameters are global parameters, they affect all imaging voxels in the same way. As a result, the ASL difference signal obtained using QUIPSS II is a quantitative measure of perfusion, lacking only a global scaling factor. In physically healthy subjects with or without major depression, there should be very little (if any) measurable differences in either M0b or blood $\mathrm{T} 1$ over a period of days.

For the present pilot study, we hypothesized: (a) Depressed responders' baseline ventral anterior cingulate (BA 25 and ventral 24) / medial prefrontal cortical (BA 32) perfusion as measured by fMRI will be greater than that of nonresponders and controls; (b) following PSD, MR perfusion in the ventral anterior cingulate/medial prefrontal cortical areas will significantly decrease only in responders.

\section{METHODS}

\section{Subjects}

Two depressed patients and one normal control participated in this study. To enter the study, depressed subjects had to meet full diagnostic criteria for current DSM-IV (Diagnostic and Statistical Manual, 4th edition) major depressive disorder (unipolar) and to have a baseline 17-item Hamilton Depression Rating Scale $($ HDRS17) $\geqslant 16$. The control subject had no psychiatric disorder based on structured interview. All subjects received the Structured Clinical Interview for DSM-IV (SCID) as well as thorough medical and laboratory evaluations as previously described (Clark et al. 1999). All subjects were physically healthy and medication free for at least two weeks at the time of the study.

Prospective subjects were excluded for contraindications to MRI or SD, significant comorbid psychiatric or substance use disorders; primary sleep disorders or irregular or deviant sleep cycle (e.g., shift work), or for any history of neurological (e.g., loss of consciousness $>$ $5 \mathrm{~min}$ ) or circulatory conditions (e.g., hypertension) which could affect sleep, EEG, brain circulation or cerebral glucose metabolism.

The UCSD Human Subjects Committee approved this study and its consent forms. All subjects had full capacity to give informed consent and signed consent forms.

\section{General Procedures}

Subjects spent an adaptation night, a baseline night, a PSD night, and a "recovery" night in the sleep laboratory, with standard polysomnographic recording montage and sleep architecture scoring procedure (Clark et al. 1999) for all nights. On the PSD night, subjects had to remain awake beginning at 3 A.M. and were closely supervised by laboratory personnel. Subjective and objective mood ratings, including the HDRS17, were administered at standard times during baseline and sleep-deprived days, during the latter part of the PSD night, and the morning after the recovery night. Clinical response was quantified by the percent decrease in the modified HDRS17 (e.g., omitting the sleep and weight loss items) from baseline to the lowest score following PSD. Remission was defined by a reduction of at least $30 \%$ in the modified HDRS17.

\section{fMRI Procedures}

At approximately 8 P.M. on baseline and sleep-deprived nights, we obtained structural and resting perfusion images on all subjects on a GE 1.5 T scanner. Subjects were instructed to relax but remain awake and motionless throughout the scans; they were fitted with soft ear plugs and positioned carefully in the head coil with comfortable supports and cushions.

At each scanning session high-resolution anatomical images of the entire brain were obtained by a Spoiled Grass (SPGR) protocol (TR: $24 \mathrm{~ms}$, TE: $5 \mathrm{~ms}$, Flip Angle: $45^{\circ}$, FOV: $240 \mathrm{~mm}$, NEX: 2). Perfusion weighted images were obtained using a spiral imaging (Li et al. 1999) refinement of the QUIPSS II (Wong et al. 1997, 1998) sequence developed at UCSD; the spiral protocol was implemented on the VA magnet by our physics team (Drs. Frank, Wong, and Buxton). Slices were chosen to cover as much of the anterior cingulate area as possible. Seven 8-mm thick axial slices with an in-plane resolution of $1.875 \mathrm{~mm}$ by $1.875 \mathrm{~mm}$ were obtained, using a gradient echo pulse sequence with the following MR parameters: TR: $3200 \mathrm{~ms}$, TE: minimum; flip angle $90^{\circ}$, FOV: $240 \mathrm{~mm}$, NEX: 1. (Because this spiral protocol is a sequence of rapidly imaged slices each tagged individually, it minimizes transit delay-based errors typical of methods that measure perfusion in multiple slices following a single tag.)

Using the AFNI (Analysis of Functional NeuroImages) 2.24g package (Cox 1997), we made the SPGR images into an anatomic brick, transformed it into Talairach space, and coregistered it with a similarly formed perfusion brick. We then viewed maps of perfusion in which each voxel was divided by the mean value of all 
intracranial perfused voxels for that scanning session. For display purposes, we used a four-color scale, ranging from yellow (225-300\% of mean perfusion and representing only about 4 to $5 \%$ of all voxels) to red (depicting minimal perfusion.) To facilitate visualization of within-subject comparisons, we used each subject's same "color boundaries" on the color scale for his or her sleep-deprived scan.

\section{RESULTS}

Figure 1 shows midsagittal views of each subject's highresolution anatomic scans. The top and bottom rows display baseline and sleep-deprived perfusion, respec- tively. From left to right are a depressed responder (50\% decrease in modified HDRS17; 41-year-old righthanded Hispanic male), a normal control (32-year-old right-handed Caucasian female), and a depressed nonresponder (32-year-old right-handed Caucasian female). (Sleep-deprived data for the normal control were unavailable for technical reasons.)

At baseline, the responder exhibited elevated perfusion in a large prefrontal area covering pregenual anterior cingulate and extending anteriorly into frontal pole and posteriorly under the corpus callosum (e.g., subgenual anterior cingulate gyrus.) The normal control's area of highest perfusion covered a markedly smaller area which did not extend nearly as far anteriorly or superiorly. The nonresponder appeared to exhibit even

\section{Perfusion Maps}
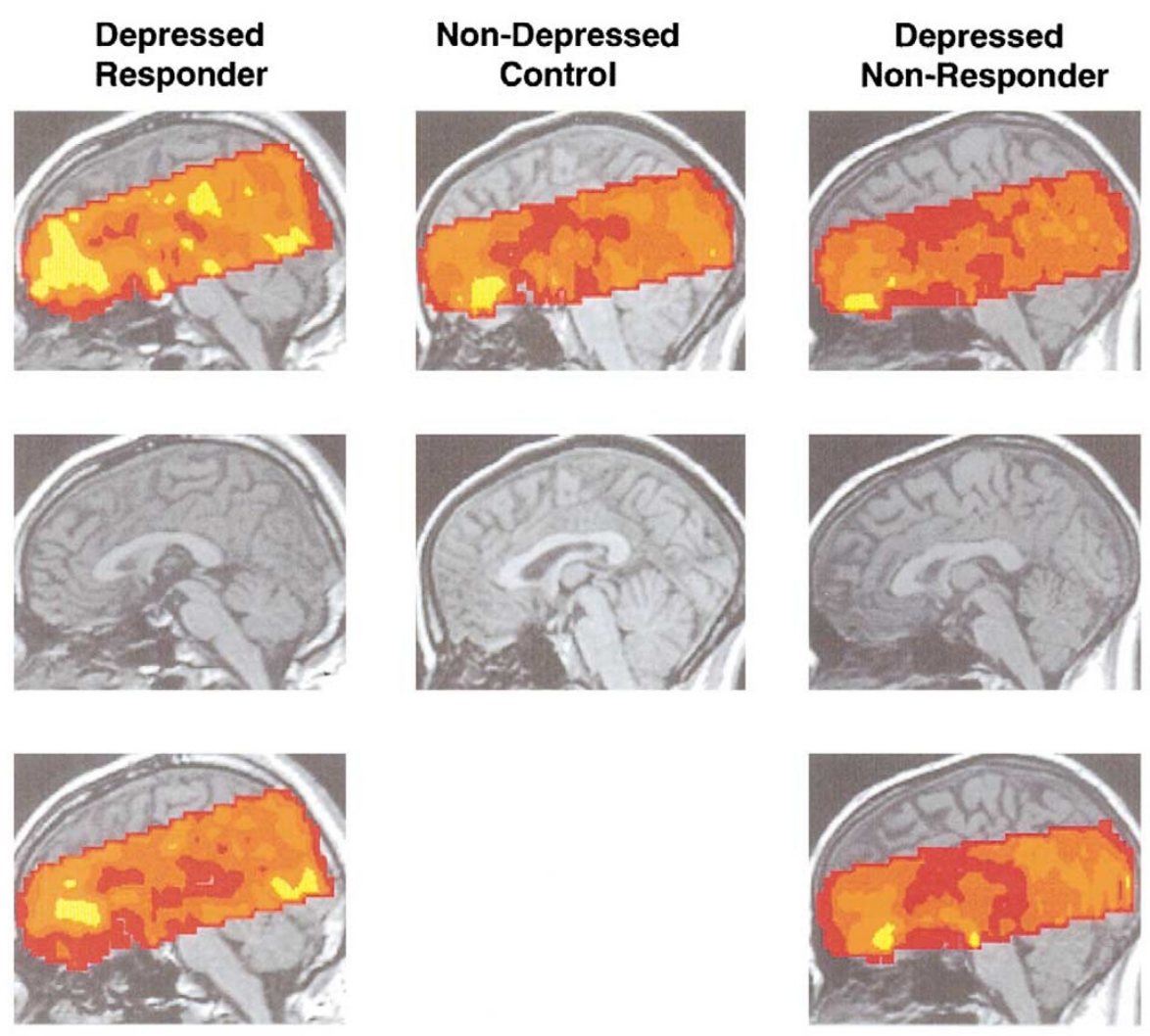
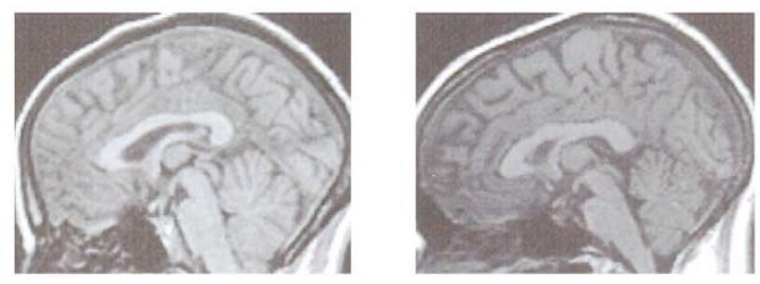

Mid-Sagittal
View

Rested

\section{Non-Depressed Control}
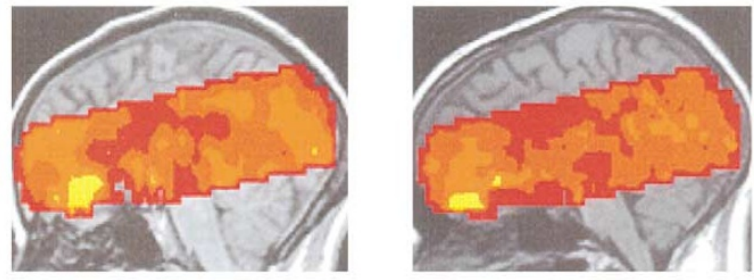

(a)

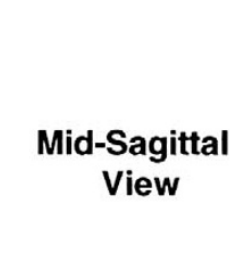

\section{Sleep \\ Deprived}

\section{Note. This responder experienced a $\mathbf{5 0 \%}$ improvement in the Modified Hamilton Depression Rating Scale following partial sleep deprivation.}

Figure 1. The images displayed are midsagittal views of each subject's high-resolution anatomic scans. The top and bottom rows display baseline (rested) and sleep-deprived perfusion, respectively. From left to right are a depressed responder, a normal control, and a depressed nonresponder.

In these perfusion maps, each voxel was divided by the mean value of all perfused voxels for that scanning session. Colors range from yellow (225-300\% of mean perfusion) to red (0-75\% of mean perfusion.) To facilitate visualization of within-subject comparisons, we used the same values for normalized perfusion "color boundaries" for each subject's baseline and sleep-deprived scans. 
lower normalized perfusion than the control subject, particularly in ventral frontal and subgenual areas.

After sleep deprivation, the responder's signal clearly decreased over much of the area of initially high normalized perfusion; in contrast, there was little change in the nonresponder's scan.

\section{DISCUSSION}

Our findings of increased baseline pregenual/subgenual anterior cingulate/medial frontal perfusion in the responder (and the obvious decrease following PSD) fit very well with previous functional neuroimaging studies of SD, particularly the PET findings of $\mathrm{Wu}$ et al. (1999) in their TSD study.

Both their study and our pilot data demonstrate: (a) baseline activity of responders greater than nonresponders in parts of BA 24a and 32; (b) baseline activity of nonresponders less than controls in ventral BA 24a and 32; (c) baseline activity of responders greater than controls in subgenual cortex; and (d) decreased activity of responders in an area including portions of BA 32 following SD (Wu et al. 1992; Ebert et al. 1991, 1994; Volk et al. 1992, 1997).

Obviously, our findings are extremely preliminary and will need to be replicated. Nonetheless, we have shown the feasibility of using MR methods to obtain high-quality quantitative perfusion measures in the primary regions of interest before and after SD. Further, we have demonstrated one approach to dealing with problems associated with multi-slice MR perfusion methods. MR quantitative perfusion methodology, (Wong et al. 1998) with its superior spatial and temporal resolution, may improve the localization of depression-associated alterations of brain function, and changes of brain function associated with treatments of depression (Gillin et al. 2001).

\section{ACKNOWLEDGMENTS}

Supported in part by NIH grants M01-RR00827, MH18399, MH30914, MHR03-54642, the VA MIRECC, the Forest Laboratories Junior Faculty Research Award, the NARSAD Young Investigator Award, and the VA Postdoctoral Fellowship in Psychiatric Research.

We would like to thank the staff of the UCSD Mental Health Clinical Research Center, the sleep laboratory, and M. J. Meloy, Ph. D. Most of all, we wish to express our gratitude to J. Christian Gillin, M.D., who has been integrally involved in this project from the time of its inception.

\section{REFERENCES}

Buchsbaum MS, Wu JC, Siegel BW, Hackett E, Trenary M, Abel L, Reynolds C (1997): Effect of sertraline on regional metabolic rate in patients with affective disorders. Biological Psychiatry 41:15-22

Clark CP, Gillin JC, Golshan S, Demodena A, Smith TL, Danowski S, Irwin M, Schuckit M (1999): Polysomnography and depressive symptoms in primary alcoholics with and without a life time diagnosis of secondary depression and in patients with primary major depression. J Affective Disorders 52:177-185

Cox R (1997): AFNI: Software for analysis and visualization of functional magnetic resonance neuroimages. Computers and Biomedical Research 29:162-173

Ebert D, Feistel H, Barocka A (1991): Effects of sleep deprivation on the limbic system and the frontal lobes in affective disorders: A study with Tc-99m- HMPAO SPECT. Psychiatry Res.Neuroimaging 40:247-251

Ebert D, Feistel H, Barocka A, Kaschka W (1994): Increased limbic blood flow and total sleep deprivation in major depression with melancholia. Psychiatry Research 55:101-109

Gillin JC (1983): The sleep therapies of depression. Progress in Neuro-psychopharmacology and Biological Psychiatry 7:351-364

Gillin JC, Buchsbaum M, Wu J, Clark C, Bunney Jr, W (2001): Sleep Deprivation as a Model Experimental Antidepressant Treatment: Findings from Functional Brain Imaging. Depression (in press)

Leibenluft E, Wehr TA (1992): Is sleep deprivation useful in the treatment of depression? Am J Psychiatry 149:159-168

Li T-Q, Takahashi A, Moseley ME, Glover GH (1999): A single-shot dual-echo spiral FAIR sequence for simultaneous measurements of CBF and oxygenation in fMRI. Abstract, International Society for Magnetic Resonance in Medicine (meeting)

Mayberg HS, Brannan SK, Mahurin RK, Jerabek PA, Brickman JS, Tekell JL, Silva A, McGinnis S, Glass TG, Martin CC, Fox PT (1997): Cingulate function in depression: a potential predictor of treatment response. NeuroReport 8:1057-1061

Ogawa S, Menon RS, Kim S, Ugurbil K (1998): On the characteristics of functional MRI of the brain. Ann Rev Biophys Biomolecular Struct 27:447-474

Volk S, Kaendler SH, Weber R, Georgi K, Maul F, Hertel A, Pflug B, Hoer G (1992): Evaluation of the effects of total sleep deprivation on cerebral blood flow using single photon emission computerized tomography. Acta Psychiatrica Scandinavica 86:478-483

Volk SA, Kaendler SH, Hertel A, Maul FD, Manoocheri R, Weber R, Georgi K, Pflug B, Hor G (1997): Can response to partial sleep deprivation in depressed patients be predicted by regional changes of cerebral blood flow? Psychiatry Res 75:67-74

Wong EC (1999): Potentials and pitfalls of arterial spin labeling based perfusion imaging techniques for MRI. In Moonen C, Bandettini PA (eds), Functional MRI. Berlin, Springer-Verlag, pp 63-69

Wong EC, Buxton RB, Frank LR (1998): Quantitative imaging of perfusion using a single subtraction (QUIPSS and QUIPSS II). Magn Reson Med 39:702-708

Wong EC, Buxton RB, Frank LR (1999): Quantitative perfusion imaging using arterial spin labeling. Neuroimaging Clin N Am 9:333-342 
Wong EC, Buxton RB, Frank LR (1997): Implementation of quantitative perfusion imaging techniques for functional brain mapping using pulsed arterial spin labeling. NMR in Biomedicine 10:237-249

Wu J, Buchsbaum MS, Gillin JC, Tang C, Cadwell S, Keator D, Fallon JH, Wiegand M, Najafi A, Klein E, Hazen K, Bunney WEJ (1999): Prediction of antidepressant effects of sleep deprivation by metabolic rates in the ventral anterior cingulate and medial prefrontal cortex. Am J Psychiat 156:1149-1158

Wu JC, Bunney WR Jr (1990): The biological basis of an antidepressant response to sleep deprivation and relapse: Review and hypothesis. Am J Psychiatry 147:14-21

Wu JC, Gillin JC, Buchsbaum MS, Hershey T, Johnson JC, Bunney WE Jr (1992): Effect of sleep deprivation on brain metabolism of depressed patients. Am J Psychiatry 149:538-543 